\title{
Non-Analytic Contributions to the Self-Energy and the Thermodynamics of Two-Dimensional Fermi Liquids
}

\author{
D. Coffey ${ }^{a}$ and K. S. Bedell ${ }^{b}$ \\ ${ }^{a}$ Center for Materials Science, MS-K765, Los Alamos National Laboratory, Los Alamos, NM \\ 87545 \\ ${ }^{b}$ Theoretical Division, MS-B262, Los Alamos National Laboratory, Los Alamos, NM 87545
}

\begin{abstract}
We calculate the entropy of a two-dimensional Fermi Liquid(FL) using a model with a contact interaction between fermions. We find that there are $T^{2}$ contributions to the entropy from interactions separate from those due to the collective modes. These $T^{2}$ contributions arise from non-analytic corrections to the real part of the selfenergy which may be calculated from the leading log dependence of the imaginary part of the self-energy through the Kramers-Kronig relation. We find no evidence of a breakdown in Fermi Liquid theory in 2D and conclude that FL in 2D are similar to 3D FL's.
\end{abstract}

05.30.Fk, 65.70+y, 67.50g

Typeset Using REVTEX 
The unusual nature of the normal state properties of the high temperature superconductors (HTS) has generated a new interest in the metallic phase of strongly correlated electronic materials. In particular, much attention has been focused on the existence [1 3] or non-existence [4] of a Fermi liquid phase for these systems in two dimensions (2D). This controversy as to the existence of $2 \mathrm{D}$ Fermi liquid(FL) is motivated by the difficulty of fitting some experimental data on the HTS materials with conventional FL expressions and also by the property of one dimensional systems that the ground state of a system of interacting fermions is a Luttinger Liquid (LL) rather than a FL. In particular this has lead to the development of the marginal Fermi Liquid (MFL) phenomenology [5,7] which has been used extensively to fit data. [9] However there is no microscopic calculation as yet which leads to a an MFL ground state.

The stability of a FL ground state has been studied extensively in the dilute limit and for weak coupling [3] in 2D. In the dilute limit it is possible to show that the particle-particle channel diagrams contribute in leading order. The presence of a two hole bound state in this channel lead to speculations that this was a possible source of the breakdown of the Fermi liquid phase. [10] It appears, though, that this bound state only gives rise to higher order corrections to the properties of the FL. In weak coupling away from half filling this stability of the FL phase of the 2D Hubbard model was also observed in the propagator renormalized fluctuation exchange approximation of Serene and Hess. [3] In this approach all of the known instabilities, superconductivity, spin and charge density waves, and the two hole bound state could occur. No evidence for a breakdown of the FL phase was observed at quarter filling.

From these studies we see that the FL phase of the Hubbard model is stable against particle-hole or particle-particle fluctuations away from half-filling. From the two-hole bound state it was shown [四] that this contributed a term of the order $|\epsilon|^{5 / 2}$ to the imaginary part of the self-energy, $\Sigma(\mathrm{p}, \epsilon)$, and from Kramers-Kronig a similar term is found in $\operatorname{Re} \Sigma(\mathrm{p}, \epsilon)$. The two hole bound states are predominantly short wavelength fluctuations, in this Letter we investigate the long wavelength fluctuations. We find that they give rise to lower order 
non-analytic corrections to FL theory than does the two hole bound state. In particular, we find that $\operatorname{Re} \delta \Sigma\left(\mathrm{p}, \xi_{p}\right) \propto \operatorname{sgn}\left(\xi_{p}\right) \xi_{p}{ }^{2}$ and that this term gives rise to a $\mathrm{T}^{2}$ correction to the specific heat, $\mathrm{C}_{V}$. What we learn from our work and that of references 1 and 2 is that the breakdown of FL theory must be more subtle than is found in any of the traditional perturbation theory approaches. The present calculation can not address the issue raised by Anderson 4,10 as to the validity of perturbation theory in $2 \mathrm{D}$ except to say that there is no indication of this from perturbation theory itself.

Apart from the question of stability of the Fermi liquid our results for the corrections to Fermi liquid theory are most surprising. In $3 \mathrm{D}$ the specific heat is known to have a $T^{3} \ln T$ correction, i.e. $\mathrm{C}_{V} \simeq \gamma \mathrm{T}+\Gamma_{3 D} \mathrm{~T}^{3} \ln \mathrm{T}$. In the 1D system the breakdown of the FL can be seen already in the $2^{\text {nd }}$ order perturbation theory where the specific heat correction is given by $\delta \mathrm{C}_{V} \simeq \Gamma_{1 D} \mathrm{~T} \ln \mathrm{T}$. (This is a clear signal that perturbation theory does not work since this is more important than the linear term). In the $2 \mathrm{D}$ case we find that $\mathrm{C}_{V} \simeq \gamma \mathrm{T}+\Gamma_{2 D}$ $\mathrm{T}^{2}+\ldots$. One might have expected this to have a $\mathrm{T}^{2} \ln \mathrm{T}$ correction by studying the $3 \mathrm{D}$ and $1 \mathrm{D}$ behavior, this in fact is not the case.

In order to determine the leading corrections to a 2D FL due to longwavelength interactions we consider a system of fermions which interact via a two-body potential as in Eq. (1).

$$
H=\sum_{\vec{p}, \sigma} \frac{|\vec{p}|^{2}}{2 m} c_{\vec{p}, \sigma}^{\dagger} c_{\vec{p}, \sigma}+\sum_{\vec{p}, \vec{q}, \alpha, \beta, \gamma, \delta} V_{\alpha, \beta, \gamma, \delta}(\vec{q}) c_{\vec{p}, \alpha}^{\dagger} c_{\vec{p}^{\prime}, \beta} c_{\vec{p}^{\prime}-\vec{q}, \gamma}^{\dagger} c_{\vec{p}+\vec{q}, \delta}
$$

Expanding in the particle-hole channel the effect of the interaction may be considered as coming from two independent channels, the symmetric(s) and the antisymmetric(a) channels corresponding to no spin exchanged and to spin 1 exchanged. Using the paramagnon model first introduced by Doniach and Engelsberg [11], for the $\vec{q}$ dependence of the interaction, the value of the interaction in the symmetric channel, $V_{s}$, is $-\mathrm{I} / 2$ and in the antisymmetric channel, $V_{a}$, is $2 I$, where $I>0$. [12] I is the the strength of the interaction and multiplied by the density of states of one spin is the paramagnon parameter, $\bar{I}$. The interaction is cutoff at $|\vec{q}|=q_{c}$. In order to compare the properties of FLs in 2D and 3D we first calculate the 
single-particle self-energy to second order in perturbation theory at zero temperature. For a 3D FL Blaizot and Friman [13] found

$$
\operatorname{Im} \Sigma(\vec{p}, \epsilon)=\frac{\pi \bar{I}^{2}}{8 v_{F}^{2} p_{F}^{2}} \operatorname{sgn}(\epsilon)\left(\left[q_{c} v_{F}-\left|\xi_{p}\right|\right] \epsilon^{2}+\frac{1}{3}|\epsilon|^{3}+\ldots\right),
$$

where $\xi_{p}=\left(p-p_{F}\right) v_{F}, p_{F}$ is the Fermi momentum, and $v_{F}=p_{F} / m$ is the Fermi velocity. The real part of $\Sigma(\vec{p}, \epsilon)$ is determined from the Kramers-Kronig relation,

$$
\operatorname{Re} \Sigma(\vec{p}, \epsilon)=\frac{1}{\pi} P \int_{-\infty}^{\infty} d \zeta \frac{\operatorname{Im} \Sigma(\vec{p}, \zeta)}{\epsilon-\zeta}
$$

and is given by

$$
\operatorname{Re} \Sigma\left(\vec{p}, \xi_{p}\right)=A_{3 D} \xi_{p}+B_{3 D} \xi_{p}^{3} \ln \left|\xi_{p}\right|+. .
$$

The $\xi_{p}^{3} \ln \left|\xi_{p}\right|$ term comes from the $|\epsilon|^{3}$ term in $\operatorname{Im} \Sigma(\vec{p}, \epsilon)$ and so it is determined by the long-wavelength scattering. In fact it has been shown by Moriya [14] that no $\xi_{p}{ }^{3} \ln \left|\xi_{p}\right|$ terms come from finite $\vec{q}$ scattering. For a $2 \mathrm{D}$ FL we find

$$
\begin{array}{r}
\operatorname{Im} \Sigma(\vec{p}, \epsilon)=\operatorname{sgn}(\epsilon) \frac{4 \bar{I}^{2}}{2 \pi N(0) v_{F}^{2}}[ \\
{\left[\xi_{p}{ }^{2}+2 \xi_{p}\left(\epsilon-\xi_{p}\right)\right) \ln \left(\max \left[\xi_{p},|\epsilon|\right]\right) \Theta\left(q_{c} v_{F}-|\epsilon|\right)} \\
\left.+\left(\epsilon-\xi_{p}\right)^{2} \ln \left(\left|\epsilon-\xi_{p}\right|\right) \Theta\left(q_{c} v_{F}-\left|\epsilon-\xi_{p}\right|\right)+. .\right] .
\end{array}
$$

for $\xi_{p}>0$. This reduces to the well-known results of Hodges et al. [15] and Bloom [16] for $\operatorname{Im} \Sigma\left(\vec{p}, \epsilon=\xi_{p}\right)$. Using the Kramers-Kronig relation again, $\operatorname{Re} \Sigma\left(\vec{p}, \xi_{p}\right)$ is

$$
\operatorname{Re} \Sigma\left(\vec{p}, \xi_{p}\right)=A_{2 D} \xi_{p}+B_{2 D} \operatorname{sgn}\left(\xi_{p}\right) \xi_{p}^{2}+\ldots \ldots
$$

Only the first term in $\operatorname{Im} \Sigma(\vec{p}, \epsilon)$ contributes to $\operatorname{Re} \Sigma\left(\vec{p}, \xi_{p}\right)$. The linear terms in $\operatorname{Re} \Sigma\left(\vec{p}, \xi_{p}\right)$ for $2 \mathrm{D}$ and $3 \mathrm{D}$ are effective mass enhancements, which come from all $\vec{q}$ s and depend on the $\vec{q}$ dependence of the interaction. Here we are concerned with the corrections to the effective mass enhancement terms. As in the case of $B_{3 D}, B_{2 D}$ is determined by long wavelength scattering. Comparing the corrections to the effective mass terms in $\operatorname{Re} \Sigma\left(\vec{p}, \xi_{p}\right)$ in $2 \mathrm{D}$ and $3 \mathrm{D}$, one sees that in 2D the correction is non-analytic and comes from the leading $\epsilon$ dependence of $\operatorname{Im} \Sigma(p, \epsilon)$ whereas in 3D the correction is analytic but comes from non-analytic terms in 
$\operatorname{Im} \Sigma(p, \epsilon)$. This difference between the leading corrections to the linear $\xi_{p}$ dependence in $2 \mathrm{D}$ and $3 \mathrm{D}$ is due solely to the different phase space. This may be seen by calculating the contribution to the spectrum in $2 \mathrm{D}$ using the equation,

$$
\Delta \epsilon_{p}=\sum_{|\vec{q}|<q_{c}}\left[1-2 f_{\vec{p}+\vec{q}}\right](\hat{p} \cdot \hat{q})^{2} V^{(2)}
$$

where $V^{(2)}$ is the coefficient of the $(\hat{p} . \hat{q})^{2}$ term in the effective quasiparticle interaction. In 3D this gives the $\xi_{p}^{3} \ln \left|\xi_{p}\right|$ dependence. [17] In contrast to $1 \mathrm{D}$, where the analogous calculation already shows that FL theory has broken down, there is no indication of a breakdown of FL theory in $2 \mathrm{D}$ to this order in perturbation theory. We now consider the thermodynamics of a 2D FL and compare the results with $3 \mathrm{D}$.

Using the RPA approximation the change in the thermodynamical potential due to interactions in Eq.(1) is given by

$$
\Delta \Omega=k_{B} T \sum_{\vec{q}, \omega_{n}}\left[\frac{3}{2}\left(\ln \left[1-I \chi\left(\vec{q}, \omega_{n}\right)\right]+I \chi\left(\vec{q}, \omega_{n}\right)\right)+\frac{1}{2}\left(\ln \left[1+I \chi\left(\vec{q}, \omega_{n}\right)\right]-I \chi\left(\vec{q}, \omega_{n}\right)\right)\right]
$$

where

$$
\chi(\vec{q}, \omega)=2 \sum_{\vec{p}} \frac{f_{\vec{p}+\vec{q}}-f_{\vec{p}}}{\omega-\left(\epsilon_{\vec{p}+\vec{q}}-\epsilon_{\vec{p}}\right)},
$$

$f_{\vec{p}}$ is the Fermi Dirac distribution function, and $\omega_{n}=2 \pi(\mathrm{n}+1) \mathrm{T}$ are Matsubara frequencies. When analytically continued to the real $\omega$ axis $\Delta \Omega$ can be easily broken up into a quasiparticle contribution, $\Delta \Omega_{q p}$, and a contribution from collective modes, $\Delta \Omega_{\text {coll.modes }}$. First we consider $\Delta \Omega_{q p}$ which is given by

$$
\begin{gathered}
\Delta \Omega_{q p}=\sum_{|\vec{q}|<q_{c}} \int_{0}^{\infty} \frac{d \omega}{\pi} n_{B}(\omega)\left(F(\vec{q}, \omega)+I \chi^{\prime \prime}(\vec{q}, \omega)\right) \\
F(\vec{q}, \omega)=\frac{3}{2} \tan ^{-1}\left[\frac{-I \chi^{\prime \prime}(\vec{q}, \omega)}{1-I \chi^{\prime}(\vec{q}, \omega)}\right]+\frac{1}{2} \tan ^{-1}\left[\frac{I \chi^{\prime \prime}(\vec{q}, \omega)}{1+I \chi^{\prime}(\vec{q}, \omega)}\right], \\
\chi(\vec{q}, \omega)=\chi^{\prime}(\vec{q}, \omega)+\imath \chi^{\prime \prime}(\vec{q}, \omega),
\end{gathered}
$$

and $n_{B}(\omega)$ is the Bose distribution function. From this the change in the entropy is 


$$
\begin{aligned}
\Delta S_{q p} & =-\left[\frac{\partial \Delta \Omega_{q p}}{\partial T}\right]_{\mu} \\
& =\sum_{|\vec{q}|<q_{c}} \int_{0}^{\infty} \frac{d \omega}{\pi}\left(\left.\frac{\partial n_{B}(\omega)}{\partial T}\right|_{\mu} F(\vec{q}, \omega)+\left.n_{B}(\omega) \frac{\partial F(\vec{q}, \omega)}{\partial T}\right|_{\mu}+\left.I \frac{\partial\left(n_{B}(\omega) \chi^{\prime \prime}(\vec{q}, \omega)\right)}{\partial T}\right|_{\mu}\right)
\end{aligned}
$$

The two terms on the right of Eq.(13) involve the temperature dependence of $\chi(\vec{q}, \omega)$ which is weak when $\mu$ is kept constant.

Calculating $\Delta S_{q p}$ for $2 \mathrm{D}$ one finds

$$
\begin{array}{r}
\Delta S_{q p}=\gamma_{2 D} \prime T+\Gamma_{2 D} T^{2}+O\left(T^{3}\right) \\
\gamma_{2 D^{\prime}}=\frac{\pi}{6 T_{F}}\left(A_{s}+A_{a}\right) \frac{q_{c}}{p_{F}} \\
\Gamma_{2 D}=\frac{\pi n}{4 T_{F}^{2}} \sum_{\lambda} \nu_{\lambda}\left(A_{\lambda}+\int_{0}^{1} d u f_{\lambda}(u)\right)
\end{array}
$$

where

$$
f_{\lambda}(u)=\frac{\left[A_{\lambda} u-\tan ^{-1}\left(\frac{A_{\lambda} u}{1-u^{2}}\right)\right]}{u^{3}},
$$

where $\lambda=\mathrm{s}$ or a, $\nu_{s}=1, \nu_{a}=3$, and

$$
A_{s}=\frac{\bar{I}}{1+\bar{I}}, A_{a}=\frac{-\bar{I}}{1-\bar{I}} .
$$

$A_{s}$ and $A_{a}$ are the scattering amplitudes in the symmetric(density) and antisymmetric(spin) channels, $\mathrm{n}$ is the density of particles, and $T_{F}=v_{F} p_{F} / 2$. The $T^{2}$ term in $\Delta S_{q p}$ comes from the non-analytic term in $\operatorname{Re} \Sigma\left(\vec{p}, \xi_{p}\right)$ as may be seen from the following argument. [18] Consider the entropy of a FL whose spectrum is given by

$$
\epsilon_{p}=\xi_{p}+\Delta \epsilon_{p}
$$

where $\Delta \epsilon_{p}$ arises from interactions. Substituting this spectrum into the expression for the entropy of a non-interacting Fermi liquid and expanding to linear order in $\Delta \epsilon_{p}$ one finds

$$
\Delta S=\left.\sum_{\vec{p}} \frac{\xi_{p}}{T} \frac{\Delta \epsilon_{p}}{T} \frac{\partial f(\epsilon)}{\partial \epsilon}\right|_{\xi_{p}}
$$

Assuming that $\Delta \epsilon_{p}$ can be expanded in a power series in $\xi_{p}$ one finds 


$$
\Delta S=N(0) k_{B} \int_{-\infty}^{\infty} \frac{\xi d \xi}{T^{2}} \frac{1}{4 \cosh ^{2} \frac{\xi}{2 T}} \sum_{n} \alpha_{n} \xi^{n}
$$

One sees that only odd $\mathrm{n}$ terms contribute to $\Delta S$ and that they lead to series of odd powers of $\mathrm{T}$. The presence of the $T^{2}$ in $\Delta S_{q p}$ clearly arises from the non-analytic nature of the correction to the spectrum in Eq.(6). The terms of $\mathrm{O}\left(T^{3}\right)$ and higher are a sum of odd powers of temperature.

Carrying out the calculations in 3D one finds that

$$
\Delta S_{q p}=\gamma_{3 D^{\prime}} T+\Gamma_{3 D} T^{3} \ln T+O\left(T^{3}\right)
$$

Another difference between $2 \mathrm{D}$ and $3 \mathrm{D}$ is that $\Gamma_{2 D}$ depends on the scattering amplitudes to all orders whereas $\Gamma_{3 D}$ involves only the second and third powers of the scattering amplitudes.

The collective mode contribution in $2 \mathrm{D}$ is

$$
\Delta S_{\text {coll.mode }}=\Gamma^{\prime \prime} T^{2}+O\left(T^{4}\right)
$$

where

$$
\Gamma^{\prime \prime}=\frac{1}{2 \pi}\left(\frac{T}{c}\right)^{2}
$$

and $\mathrm{c}$ is the velocity of the collective mode given by

$$
c=v_{F} \frac{1+\bar{I}}{\sqrt{1+2 I}}
$$

The collective mode spectrum does not contain any log dependence on $|\vec{q}|$ and so there is

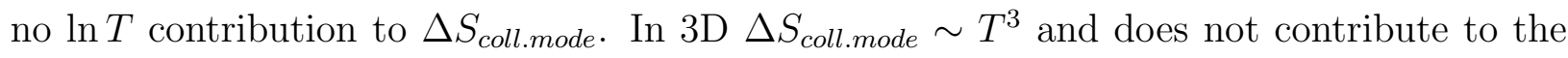
$T^{3} \ln T$ corrections except to change the cutoff of the logarithmic temperature dependence.

Collecting $\Delta S_{q p}$ and $\Delta S_{\text {coll.mode }}$ together one sees that $\Delta S$ is a power series in temperature in $2 \mathrm{D}$. In particular there are no $\ln T$ terms in $\Delta S$ which implies that, at least to leading order, quasiparticle damping effects do not contribute to thermodynamic properties in this approximation where the propagators are unrenormalised. The effect of finite quasiparticle lifetimes on the entropy may be estimated with Eq.(23) of ref. [19 and is given by 


$$
\Delta S_{\text {damp }}=\sum_{\vec{p}} \int_{-\infty}^{\infty} d \epsilon \frac{\partial f(\epsilon)}{\partial T} G(\lambda(\vec{p}, \epsilon))
$$

where

$$
G(\lambda(\vec{p}, \epsilon))=\frac{\lambda(\vec{p}, \epsilon)}{1+\lambda(\vec{p}, \epsilon)^{2}}-\tan ^{-1}(\lambda(\vec{p}, \epsilon))
$$

and

$$
\lambda(\vec{p}, \epsilon)=-\frac{\operatorname{Im} \Sigma(\vec{p}, \epsilon)}{\operatorname{Re} \Sigma(\vec{p}, \epsilon)}
$$

The functional form of the integrand in Eq. (25) is very complicated and analytic evaluation is intractable. So we content ourselves with an estimate. $G(\lambda(\vec{p}, \epsilon)$ is a smooth function of $\lambda(\vec{p}, \epsilon)$ which goes as $\lambda(\vec{p}, \epsilon)^{3}$ for small $\lambda(\vec{p}, \epsilon)$ and is a constant for large $\lambda(\vec{p}, \epsilon)$. In order to get an estimate of $\Delta S_{\text {damp }}$ we assume that $G \sim \lambda(\vec{p}, \epsilon)^{3}$ for all values of $\epsilon$. Since there is no contribution to the integral for large values of $|\lambda(\vec{p}, \epsilon)|$, this is clearly an overestimate. With these approximations one finds $\Delta S_{\text {damp }} \sim T^{7} \ln ^{3} T$ which is higher order in temperature than the $T^{2}$ corrections found above. Lifetime effects lead to higher order effects in the thermodynamics than $T^{2}$ so that they are much less important in thermodynamics than a calculation of $\Sigma(\vec{p}, \epsilon)$ would suggest.

Since we have used a particle-hole expansion in the symmetric and antisymmetric channels our results may be easily extended to Landau's Fermi liquid theory by considering Eq.(1) to describe quasiparticles with an effective mass interacting via an effective interaction $f\left(\vec{p}, \vec{p}^{\prime}\right)$ which can be decomposed into two channels, $f_{s}\left(\vec{p}, \vec{p}^{\prime}\right)$ and $f_{a}\left(\vec{p}, \vec{p}^{\prime}\right)$. This effective interaction is a long wavelength limit of the particle-hole irreducible four-point vertex and so describes long wavelength properties. This leads to somewhat more complicated expressions when $V_{s}$ and $V_{a}$ are substituted for by $f_{s}\left(\vec{p}, \vec{p}^{\prime}\right)$ and $f_{a}\left(\vec{p}, \vec{p}^{\prime}\right)$. The Landau functions are functions of the variable $s=\frac{\omega}{q v_{F}}$ and may be expressed as coefficients in a series of Legendre polynomials in which $s$ is the argument. These coefficients are the Landau parameters.

The present calculation indicates that a $2 \mathrm{D}$ FL is very similar to the $3 \mathrm{D}$ case and that any breakdown of the FL in 2D has to arise from effects which are more subtle than those which 
give the leading corrections to FL theory in $3 \mathrm{D}$. The logarithmic dependence in $\operatorname{Im} \Sigma(\vec{p}, \epsilon)$ allow us to keep track of the contribution to the thermodynamic properties from lifetime effects. We find that in spite of the $\xi_{p}{ }^{2} \ln \xi_{p}$ dependence of the relaxation time in $2 \mathrm{D}$ their contributions are higher order in $\mathrm{T}$ than the contributions to the quasiparticle spectrum.

The authors would like to thank C. J. Pethick for several important contributions to this work. DC acknowledges the support of the Center for Materials Science through the Program in Correlated Electron Theory. This work was supported by the US Department of Energy. 


\section{REFERENCES}

1 J. R. Engelbrecht and M. Randeria, Phys. Rev. Lett. 65, 1032 (1990) and Phys. Rev. B 45, 12419 (1992).

2 H. Fukuyama, Y. Hasegawa and O. Narikiyo, J. Phys. Soc. Jap. 60, 2013 (1991).

3 J. W. Serene and D. W. Hess, Phys. Rev. B 44, 3391 (1991).

$4 \quad$ P. W. Anderson, Phys. Rev. Lett. 64, 1839 (1990).

5 C. M. Varma, P. Littlewood, S. Schmitt-Rink, E. Abrahams and A. E. Ruckenstein, Phys. Rev. Lett. 63, 1996 (1989).

$6 \quad$ G. Zimanyi and K. S. Bedell, Phys. Rev. Lett. 66, 228 (1991).

$7 \quad$ S. Schmitt-Rink, C. M. Varma and A. E. Ruckenstein, Phys. Rev. Lett. 63, 445 (1989).

$8 \quad$ P. C. Stamp, Phys. Rev. Lett. 68, 2180 and 3938 (1992).

9 B. Batlogg in High Temperature Superconductivity: Proceedings of the Los Alamos Symposium 1989, edited by K. S. Bedell, D. Coffey, D. E. Meltzer, D. Pines and J. R. Schrieffer, (Addison-Wesley, Redwood City, California, 1990), page 37.

10 P. W. Anderson, Phys. Rev. Lett. 65, 2306 (1990).

11 S. Doniach and S. Engelsberg, Phys. Rev. Lett., 17, 750 (1966).

12 W. F. Brinkman and S. Engelsberg, Phys. Rev. 169, 417 (1968).

13 J. P. Blaizot and B. L. Friman, Nucl. Phys. A372, 69 (1981) .

14 T. Moriya, Phys. Rev. Lett. 24, 1433 (1970) and T. Moriya and T. Kato J. Phys. Jap. Soc. 31, 1016 (1971).

15 C. Hodges, H. Smith and J.W. Wilkins, Phys. Rev. 4, 302 (1971).

16 P. Bloom, Phys. Rev. B 12, 125 (1975). 
17 G. Baym and C. J. Pethick, in "The Physics of Liquid and Solid Helium", edited by K. H. Bennemann and J. B. Ketterson (Wiley, New York, 1978) Part II, Eq.(1.4.69), page 102.

18 C. J. Pethick, (private communication).

19 G. M. Carneiro and C. J. Pethick, Phys. Rev. B 11, 1106 (1977). 